\title{
Update in Hospital Medicine: Evidence You Should Know
}

\author{
Kathleen M. Finn, MD*, Jeffrey L. Greenwald, MD
}

Clinician Educator Service, Department of Medicine, Massachusetts General Hospital, Harvard Medical School, Boston, Massachusetts.

BACKGROUND: The practice of hospital medicine is complex, and the number of clinical publications each year continues to grow. To maintain best practice it is necessary for hospitalists to stay abreast of the literature, but difficult to accomplish due to time. The annual Society of Hospital Medicine meeting offers a plenary session on Updates in Hospital Medicine. This article is a summary of those papers presented at the meeting.

METHODS: We reviewed articles published between January 2014 and January 2015 in the leading medical journals, searching for papers with good methodological quality, the potential to change practice, and papers that are thought provoking. The authors collectively agreed on 14 articles. The findings, cautions, and implications are discussed for each paper.

RESULTS: Key findings include: a novel neprilysin inhibitor and angiotensin receptor blocker combination drug reduces mortality in patients with heart failure; the concern for acute kidney injury after venous contrast may be overstated; the Confusion Assessment Method Severity score is an important tool for prognostication in delirious patients; ramelteon shows promise for lowering incident delirium among elderly medical patients; polyethylene glycol appears effective in rapidly resolving hepatic encephalopathy; cirrhotic patients on a nonselective $\beta$-blocker have increased mortality after they develop spontaneous bacterial peritonitis; current guidelines regarding prophylaxis against venous thromboembolism (VTE) in medical inpatients likely result in nonbeneficial use of medications; from a safety and efficacy perspective, direct oral anticoagulants perform quite well against conventional therapies in patients with VTE and atrial fibrillation, including in elderly populations; 2 new once-weekly antibiotics, dalbavancin and oritivancin, approved for skin and soft tissue infections, appear noninferior to vancomycin; offering family members of a patient undergoing cardiopulmonary resuscitation the opportunity to observe has durable impact on longterm psychological outcomes.

CONCLUSIONS: This update reviews key clinical articles published in 2014, selected by the authors for their methodological quality and potential for changing the practice of inpatient physicians. All of these articles add to the body of inpatient medical knowledge and contribute to the debate on best practices. Journal of Hospital Medicine 2015;10:817826. (C) 2015 Society of Hospital Medicine
Keeping up with the medical literature in a field as broad as hospital medicine is a daunting task. In 2014 alone, there were over 9200 articles published in top-tier internal medicine journals. ${ }^{1}$ The authors have selected articles from among these top journals using a nonsystematic process that involved reviewing articles brought to their attention via colleagues, literature searches, and online services. The focus was to identify articles that would be of importance to the field of hospital medicine for their potential to be practice changing, provocative, or iconoclastic. After culling through hundreds of titles and abstracts, 46 articles were reviewed by both authors in full text, and ultimately 14 were selected for presentation here. Table 1 summarizes the key points.

\footnotetext{
*Address for correspondence and reprint requests: Kathleen M. Finn, MD, Clinician Educator Service, 50 Staniford Street, 5th Floor, Boston, MA 02114; Telephone: 617-643-4053; Fax: 617-643-1781;

E-mail: kfinn@partners.org
}

This article was published online on 9 September 2015. The title was changed. This notice is included in the online and print versions to indicate that both have been corrected 4 December 2015 .

Additional Supporting Information may be found in the online version of this article.

Received: June 4, 2015; Revised: August 3, 2015;

Accepted: August 17, 2015

2015 Society of Hospital Medicine DOI 10.1002/jhm.2476

Published online in Wiley Online Library (Wileyonlinelibrary.com).

\section{AN APPROACHING PARADIGM SHIFT IN THE TREATMENT FOR HEART FAILURE}

McMurray J, Packer M, Desai A, et al. Angiotensinneprilysin inhibition versus enalapril in heart failure. N Engl J Med. 2014;371:993-1004.

\section{Background}

The last drug approved by the Food and Drug Administration (FDA) for heart failure (HF) was 10 years ago. ${ }^{2}$ The new PARADIGM (Prospective Comparison of ARNI With ACEI to Determine Impact on Global Mortality and Morbidity in Heart Failure) heart failure study comparing a novel combination drug of a neprilysin inhibitor and angiotensin receptor blocker (ARB) to an angiotensin-converting enzyme (ACE) inhibitor has cardiologists considering a possible change in the HF treatment algorithm. Neprilysin is a naturally occurring enzyme that breaks down the protective vasoactive peptides (brain natriuretic peptide, atrial natriuretic peptide, and bradykinin) made by the heart and the body in HF. These vasoactive peptides function to increase vasodilation and block sodium and water reabsorption. This novel neprilysin inhibitor extends the life of these vasoactive peptides, thus enhancing their effect. By inhibiting both neprilysin and the renin-angiotensin system, there should be additional improvement in HF management. The neprilysin inhibitor was combined with an ARB 


\author{
TABLE 1. Summary of Key Points \\ 1. Now that neprolysin inhibitors are approved by the FDA, hospitalists will see them prescribed as an alternative to ACE-inhibitors given their impressive benefits in cardiovascular mortality and heart failure hospitalizations. \\ 2. Current evidence suggests that intravenous contrast given with CT scans may not significantly alter the incidence of acute kidney injury, its associated mortality, or the need for hemodialysis. \\ 3. The CAM-S score is an important tool for prognostication in delirious patients. Those patients with high CAM-S scores should be considered for goals of care conversations. \\ 4. The melatonin agonist, ramelteon, shows promise for lowering incident delirium among elderly medical patients, though larger trials are still needed. \\ 5. Polyethylene glycol may be an excellent alternative to lactulose for patients with acute hepatic encephalopathy once larger studies are done, as it is well tolerated and shows faster resolution of symptoms. \\ 6. Nonselective $\beta$-blockers should no longer be offered to cirrhotic patients after they develop spontaneous bacterial peritonitis, as they are associated with increased mortality and acute kidney injury. \\ 7. Current guidelines regarding prophylaxis against VTE in medical inpatients likely result in nonbeneficial use of medications for this purpose. It remains unclear which high-risk populations do benefit from pharmacologic \\ prophylaxis. \\ 8. DOACs are as effective and are safer than conventional therapy for treatment of VTE, though they are not recommended in patients with GFR $<30 \mathrm{~mL} / \mathrm{min}$. \\ 9. DOACs are more effective and are safer (though they may increase risk of gastrointestinal bleeding) than conventional therapy in patients with AF. \\ 10. DOACs are as safe and more effective than conventional therapy in elderly patients with VTE or AF, being mindful of dosing recommendations in this population. \\ 11. Two new once-weekly antibiotics, dalbavancin and oritavancin, approved for skin and soft tissue infections, appear noninferior to vancomycin and have the potential to shorten hospitalizations and, in doing so, may \\ decrease cost. \\ 12. Offering family members of a patient undergoing CPR the opportunity to observe has durable impact on meaningful short- and long-term psychological outcomes. Clinicians should strongly consider making this offer.
}

NOTE: Abbreviations: ACE, angiotensin-converting enzyme; AF, atrial fibrillation; CAM, Confusion Assessment Method; CAM-S score, Confusion Assessment Method Severity score; CPR, cardiopulmonary resuscitation; CT, computed tomography; DOAC, direct oral anticoagulant; FDA, Food and Drug Administration; GFR, glomerular filtration rate; VTE, venous thromboembolism.

instead of an ACE inhibitor because of significant angioedema seen in earlier phase trials when combined with an ACE inhibitor. This is believed related to increases in bradykinin due to both agents.

\section{Findings}

In this multicenter, blinded, randomized trial, over 10,000 patients with known HF (ejection fraction $<35 \%$, New York Heart Association class II or higher) went through 2 run-in periods to ensure tolerance of both enalapril and the study drug, a combination of a neprilysin inhibitor and valsartan (neprilysin-I/ARB). Eventually 8442 patients underwent randomization to either enalapril (10 mg twice a day) or neprilysin-I/ARB (200 mg twice a day). The primary outcome was a combination of cardiovascular mortality and heart failure hospitalizations. The trial was stopped early at 27 months because of overwhelming benefit with neprilysin-I/ARB $(21.8 \%$ vs $26.5 \%$; $P<0.001)$. There was a $20 \%$ reduction specifically in cardiovascular mortality $(13.3 \%$ vs $16.5 \%$; hazard ratio $[\mathrm{HR}]: 0.80 ; P<0.001)$. The number needed to treat (NNT) was 32 . There was also a $21 \%$ reduction in the risk of hospitalization $(P<0.001)$. More patients with neprilysin-I/ARB had symptomatic hypotension (14\% vs $9.2 \% ; P<0.001)$ but patients on the ACE inhibitor experienced more cough, hyperkalemia, and increases in their serum creatinine.

\section{Cautions}

There are 2 reasons clinicians may not see the same results in practice. First, the trial was stopped early, which can sometimes exaggerate benefits. ${ }^{3}$ Second, the 2 run-in periods eliminated patients who could not tolerate the medications at the trial doses. Additionally, although the study's authors were independent, the trial was funded by a pharmaceutical company.

\section{Implications}

This new combination drug of a neprilysin inhibitor and valsartan shows great promise at reducing cardiovascular mortality and hospitalizations for heart fail- ure compared to enalapril alone. Given the high morbidity and mortality of heart failure, having a new agent in the treatment algorithm will be useful to patients and physicians. The drug was just approved by the FDA in July 2015 and will likely be offered as an alternative to ACE inhibitors.

\section{VENOUS CONTRAST-INDUCED NEPHROTOXICITY: IS THERE REALLY A RISK?}

McDonald J, McDonald R, Carter R, et al. Risk of intravenous contrast material-mediated acute kidney injury: a propensity score-matched study stratified by baseline-estimated glomerular filtration rate. Radiology. 2014;271(1):65-73.

McDonald R, McDonald J, Carter R, et al. Intravenous contrast material exposure is not an independent risk factor for dialysis or mortality. Radiology. 2014;273(3):714-725.

\section{Background}

It is a common practice to withhold intravenous contrast material from computed tomography (CT) scans in patients with even moderately poor renal function out of concern for causing contrast-induced nephropathy (CIN). Our understanding of CIN is based largely on observational studies and outcomes of cardiac catheterizations, where larger amounts of contrast are given intra-arterially into an atherosclerotic aorta. ${ }^{4}$ The exact mechanism of injury is not clear, possibly from direct tubule toxicity or renal vasoconstriction. ${ }^{5}$ CIN is defined as a rise in creatinine $>0.5 \mathrm{mg} / \mathrm{dL}$ or $>25 \%$ rise in serum creatinine 24 to 48 hours after receiving intravenous contrast. Although it is usually self-limited, there is concern that patients who develop CIN have an increase risk of dialysis and death. ${ }^{6}$ In the last few years, radiologists have started to question whether the risk of CIN is overstated. A recent meta-analysis of 13 studies demonstrated a similar likelihood of acute kidney injury in patients regardless 
of receiving intravenous contrast. ${ }^{7}$ If the true incidence of CIN after venous contrast is actually lower, this raises the question of whether we are unnecessarily withholding contrast from CTs and thereby reducing their diagnostic accuracy. Two 2014 observational studies provide additional evidence that the concern for CIN may be overstated.

\section{Findings}

The 2 Mayo Clinic studies used the same database. They looked at all patients who underwent a contrastenhanced or unenhanced thoracic, abdominal, or pelvic CT between January 2000 and December 2010 at the Mayo Clinic. After limiting the data to patients with pre- and post-CT creatinine measurements and excluding anyone on dialysis, with preexisting acute kidney injury, or who had received additional contrast within 14 days, they ended up with 41,229 patients, mostly inpatients. All of the patients were assigned propensity scores based on risk factors for the development of CIN and whether they would likely receive contrast. The patients were then subdivided into 4 renal function subgroups based on estimated glomerular filtration rate (eGFR). The patients who received contrast were matched based on their propensity scores to those who did not received contrast within their eGFR subgroups. Unmatched patients were eliminated, leaving a cohort of 12,508 matched patients. The outcome of the first article was acute kidney injury (AKI) defined as a rise in creatinine $>0.5 \mathrm{mg} /$ $\mathrm{dL}$ at 24 to 48 hours. Though AKI rose with worsening eGFR subgroups (eGFR > $90[1.2 \%]$ vs eGFR < $30[14 \%])$, the rates of AKI were the same regardless of contrast exposure. There was no statistical difference in any of the eGFR subgroups. The second study looked at important clinical outcomes-death and the need for dialysis. There was no statistical difference for emergent dialysis (odds ratio [OR]: 0.96, $P=0.89$ ) or 30 -day mortality (HR: $0.97 ; P=0.45$ ) regardless of whether the patients received contrast or not.

\section{Cautions}

In propensity matching, unmeasured confounders can bias the results. However, the issue of whether venous contrast causes CIN will unlikely be settled in a randomized controlled trial. For patients with severe renal failure $($ eGFR $<30)$, there were far fewer patients in this subgroup, making it harder to draw conclusions. The amount of venous contrast given was not provided. Finally, this study evaluated intravenous contrast for CTs, not intra-arterial contrast.

\section{Implications}

These 2 studies raise doubt as to whether the incidence of AKI after contrast-enhanced CT can be attributed to the contrast itself. What exactly causes the rise in creatinine is probably multifactorial includ- ing lab variation, hydration, blood pressure changes, nephrotoxic drugs, and comorbid disease. In trying to decide whether to obtain a contrast-enhanced CT for patients with chronic kidney dysfunction, these studies provide more evidence to consider in the decisionmaking process. A conversation with the radiologist about the benefits gained from using contrast in an individual patient may be of value.

\section{PREVENTION AND PROGNOSIS OF INPATIENT DELIRIUM}

Hatta K, Yasuhiro K, Wada K, et al. Preventive effects of ramelteon on delirium: a randomized placebo controlled trial. JAMA Psych. 2014;71(4):397-403.

A new melatonin agonist dramatically improves delirium incidence.

\section{Background}

Numerous medications and therapeutic approaches have been studied to prevent incident delirium in hospitalized medical and surgical patients with varying success. Many of the tested medications also have the potential for significant undesirable side effects. An earlier small trial of melatonin appeared to have impressive efficacy for this purpose and be well tolerated, but the substance is not regulated by the FDA. ${ }^{8}$ Ramelteon, a melatonin receptor agonist, is approved by the FDA for insomnia, and authors hypothesized that it, too, may be effective in delirium prevention.

\section{Findings}

This study was a multicenter, single-blinded, randomized controlled trial of the melatonin-agonist ramelteon versus placebo in elderly patients admitted to the hospital ward or ICU with serious medical conditions. Researchers excluded intubated patients or those with Lewy body dementia, psychiatric disorders, and severe liver disease. Patients received either ramelteon or placebo nightly for up to a week, and the primary end point was incident delirium as determined by a blinded observer using a validated assessment tool. Sixty-seven patients were enrolled. The baseline characteristics in the arms of the trial were similar. In the placebo arm, 11 of 34 patients (32\%) developed delirium during the 7-day observation period. In the ramelteon arm, 1 of $33(3 \%)$ developed delirium $(P=0.003)$. The rate of drug discontinuation was the same in each arm.

\section{Cautions}

This study is small, and the single-blinded design (the physicians and patients knew which group they were in but the observers did not) limits the validity of these results, mandating a larger double-blinded trial.

\section{Implications}

Ramelteon showed a dramatic impact on preventing incident delirium on elderly hospitalized patients with serious medical conditions admitted to the ward or 
intensive care unit (ICU) (nonintubated) in this small study. If larger trials concur with the impact of this well-tolerated and inexpensive medication, the potential for delirium incidence reduction could have a dramatic impact on how care for delirium-vulnerable patients is conducted as well as the systems-level costs associated with delirium care. Further studies of this class of medications are needed to more definitively establish its value in delirium prevention.

\section{THE CONFUSION ASSESSMENT METHOD SEVERITY SCORE CAN QUANTIFY PROGNOSIS FOR DELIRIOUS MEDICAL INPATIENTS}

Innoye SK, Kosar CM, Tommet D, et al. The CAM-S: development and validation of a new scoring system for delirium in 2 cohorts. Ann Intern Med. 2014;160:526533.

\section{Background}

Delirium is common in hospitalized elderly patients, and numerous studies show that there are both shortand long-term implications of developing delirium. Using well studied and validated tools has made identifying delirium fairly straightforward, yet its treatment remains difficult. Additionally, differentiating which patients will have a simpler clinical course from those at risk for a more morbid one has proved challenging. Using the Confusion Assessment Method (CAM), both in its short (4-item) and long (10-item) forms, as the basis for a prognostication tool, would allow for future research on treatment to have a scale against which to measure impact, and would allow clinicians to anticipate which patients were more likely to have difficult clinical courses.

\section{Findings}

The CAM Severity (CAM-S) score was derived in 1219 subjects participating in 2 ongoing studies: 1 included high-risk medical inpatients 70 years old or older, and the other included similarly aged patients undergoing major orthopedic, general, or vascular surgeries. Outcomes data were not available for the surgical patients. The CAM items were rated as either present/absent or absent/mild/severe, depending on the item, with an associated score attached to each item such that the 4-item CAM had a score of 0 to 7 and the 10-item CAM 0 to 19 (Table 2). Clinical outcomes from the medical patients cohort showed a dose response with increasing CAM-S scores with respect to length of stay, adjusted cost, combined 90day end points of skilled nursing facility placement or death, and 90-day mortality. Specifically, for patients with a CAM-S (short form) score of 5 to 7, the 90day rate of death or nursing home residence was $62 \%$, whereas the 90 -day postdischarge mortality rate was $36 \%$.

\begin{tabular}{llc}
\hline \multicolumn{2}{l}{ TABLE 2. The Four-Item Confusion Assessment } \\
Method and Confusion Assessment Method Severity \\
Score & \multicolumn{2}{c}{ The CAM-S } \\
\hline The CAM & Absent & 0 \\
\hline Acute onset with fluctuating course & Present & 1 \\
& Absent & 0 \\
Inattention or distractability & Mild & 1 \\
& Severe & 2 \\
Disorganized thinking, illogical or unclear ideas & Absent & 0 \\
& Mild & 1 \\
Alteration of consciousness & Severe & 2 \\
& Absent & 0 \\
Total & Mild & 0 \\
& Severe & 2 \\
& & $0-7$ \\
\hline
\end{tabular}

NOTE: To diagnose delirium using the 4-item CAM, one needs to identify both of the top 2 elements and 1 or both of the bottom 2. Each present element is scored using the severity rating listed in the CAM-S column to create a sum total. The 10-item CAM additionally includes assessment of orientation, memory, perceptual disturbance, psychomotor agitation and retardation, and sleep disturbances. Each of these additional elements are assigned scores as in the 4-item CAM-S for possible scores of 0 to 17. Abbreviations: CAM, Confusion Assessment Method; CAM-S, Confusion Assessment Method Severity.

\section{Cautions}

The CAM-S, like the CAM, may work less well in patients with hypoactive delirium. This scale has been applied in a surgical cohort, but study outcomes were not presented in this article. This absence limits our ability to apply these results to a surgical population presently.

\section{Implications}

This study demonstrates that in medical inpatients, the CAM-S is effective for prognostication. Moreover, the study points out that high-scoring patients on the CAM-S have quite poor prognoses, with more than one-third dying by 3 months. This finding suggests that an important use of the CAM-S is to identify patients about whom goals of care discussions should be held and end-of-life planning initiated if not previously done.

\section{GET EXCITED ABOUT HEPATIC ENCEPHALOPATHY AGAIN-A NEW POSSIBLE TREATMENT}

Rahimi R, Singal A, Cuthbert J, et al. Lactulose vs polyethylene glycol 3350-electrolyte solution for treatment of overt hepatic encephalopathy. The HELP randomized clinical trial. JAMA Intern Med. 2014;174(11):1727-1733.

\section{Background}

Lactulose has been the principle treatment for acute hepatic encephalopathy (HE) since $1966 .{ }^{9}$ It theoretically works by lowering the $\mathrm{pH}$ of the colon and trapping ammonia as ammonium, which is then expelled. Alternatively, it may simply decrease transit time through the colon. In fact, earlier treatments for HE were cathartics such as magnesium salts. Unfortunately $20 \%$ tp $30 \%$ of 
patients are poor responders to lactulose, and patients do not like it. This new study tests whether a modern-day cathartic, polyethylene glycol, works as well as lactulose.

\section{Findings}

In this unblinded, randomized controlled trial, patients presenting to the emergency department with acute HE were assigned to either lactulose 20 to $30 \mathrm{~g}$ for a minimum of 3 doses over 24 hours or $4 \mathrm{~L}$ of polyethylene glycol (PEG) over 4 hours. The2 groups were similar in severity and etiology of liver disease. Patients were allowed to have received 1 dose of lactulose given in the emergency department prior to study enrollment. They were excluded if taking rifaximin. The primary outcome was improvement in the hepatic encephalopathy scoring algorithm (HESA) by 1 grade at 24 hours. ${ }^{10}$ The algorithm scores HE from 0 (no clinical findings of $\mathrm{HE}$ ) to 5 (comatose). Initial mean HESA scores in the 2 groups were identical (2.3).

In the lactulose group, 13/25 (52\%) improved by at least 1 HESA score at 24 hours. Two patients $(8 \%)$ completely cleared with a HESA score of 0 . In comparison, 21/23 (91\%) in the PEG group improved at 24 hours, and 10/23 (43\%) had cleared with a HESA score of $0(P<0.01)$. The median time to HE resolution was 2 days in the lactulose group compared with 1 day in the PEG group $(P=0.01)$. There were no differences in serious adverse events. The majority $(76 \%)$ of the PEG group received the full 4 L of PEG.

\section{Cautions}

The main limitations of the trial were the small sample size, that it was a single-center study, and the fact it was unblinded. Additionally, $80 \%$ of the PEG group received 1 dose of lactulose prior to enrollment. Statistically, more patients in the PEG group developed hypokalemia, which can worsen HE. Therefore, if PEG is used for acute HE, potassium will need to be monitored.

\section{Implications}

The results are intriguing and may represent a new possible treatment for acute HE once larger studies are done. Interestingly, the ammonia level dropped further in the lactulose group than the PEG group, yet there was more cognitive improvement in the PEG group. This raises questions about the role of ammonia and catharsis in HE. Although lactulose and rifaximin continue to be the standard of care, cathartics may be returning as a viable alternative.

\section{SHOULD $\beta$-BLOCKERS BE STOPPED IN PATIENTS WITH CIRRHOSIS WHEN SPONTANEOUS BACTERIAL PERITONITIS OCCURS?}

Mandorfer M, Bota S, Schwabi P, et al. Nonselective beta blockers increase risk for hepatorenal syndrome and death in patients with cirrhosis and spontaneous bacterial peritonitis. Gastroenterology. 2014;146:16801690.

\section{Background}

Nonselective $\beta$-blockers (NSBBs) are considered "the aspirin of hepatologists," as they are used for primary and secondary prevention of variceal bleeds in patients with cirrhosis. ${ }^{11}$ Since the 1980 s, their benefit in reducing bleeding risk has been known, and more recently there has been evidence that they may reduce the risk of developing ascites in patients with compensated cirrhosis. Yet, there has been some contradictory evidence suggesting reduced survival in patients with decompensated cirrhosis and infections on NSBBs. This has led to the "window hypothesis" of NSBBs in cirrhosis, where NSBBs are beneficial only during a certain window period during the progression of cirrhosis. ${ }^{12}$ Early on in cirrhosis, before the development of varices or ascites, NSBBs have no benefit. As cirrhosis progresses and portal hypertension develops, NSBBs play a major role in reducing bleeding from varices. However, in advanced cirrhosis, NSBBs may become harmful. In theory, they block the body's attempt to increase cardiac output during situations of increased physiologic stress, resulting in decreased mean arterial pressure and perfusion. This, in turn, causes end-organ damage and increased risk of death. When exactly this NSBB window closes is unclear. A 2014 study suggests the window should close when patients develop spontaneous bacterial peritonitis (SBP).

\section{Findings}

This retrospective study followed 607 consecutive patients seen at a liver transplant center in Vienna, Austria, from 2006 to 2011. All of the patients were followed from the time of their first paracentesis. They were excluded if SBP was diagnosed during the first paracentesis. Patients were grouped based on whether they took an NSBB. As expected, more patients on an NSBB had varices $(90 \%$ vs $62 \% ; P<0.001)$ and a lower mean heart rate (77.5 vs 83.9 beats/minute; $P<0.001)$. However, the 2 groups were similar in mean arterial pressure, systolic blood pressure, Model for End-Stage Liver Disease score (17.5), Childs Pugh Score (CPS) $(50 \%$ were C), and in the etiology of cirrhosis $(55 \%$ were from alcoholic liver disease). They followed the patients for development of SBP. The primary outcome was transplant-free survival. For the patients who never developed SBP, there was a $25 \%$ reduction in the risk of death for those on an NSBB adjusted for varices and CPS stage $(\mathrm{HR}=0.75$, $P=0.027)$. However, for the 182 patients who developed SBP, those on an NSBB had a $58 \%$ increase risk of death, again adjusted for varices and CPS stage $(\mathrm{HR}=1.58, P=0.014)$. Among the patients who developed SBP, there was a higher risk of hepatorenal syndrome (HRS) within 90 days for those on an NSBB 
( $24 \%$ vs $11 \%, P=0.027$ ). Although the mean arterial pressures (MAP) had been similar in the 2 groups before SBP, after the development of SBP, those on an NSBB had a significantly lower MAP (77.2 vs $82.6 \mathrm{~mm} \mathrm{Hg}, P=0.005$ ).

\section{Cautions}

This is a retrospective study, and although the authors controlled for varices and CPS, it is still possible the 2 groups were not similar. Whether patients were actually taking the NSBB is unknown, and doses of the NSBB were variable.

\section{Implications}

This study provides more evidence for the NSBB window hypothesis in the treatment of patients with cirrhosis. It suggests that the window on NSBB closes when patients develop SBP, as NSBBs appear to increase mortality and the risk of HRS. Thus, NSBB therapy should probably be discontinued in cirrhotic patients developing SBP. The question is for how long? The editorial accompanying the article says permanently. ${ }^{13}$

\section{VTE PROPHYLAXIS FOR MEDICAL INPATIENTS: IS IT A THING OF THE PAST?}

Flanders SA, Greene T, Grant P, et al. Hospital performance for pharmacologic venous thromboembolism prophylaxis and rate of venous thromboembolism. A cohort study. JAMA Intern Med. 2014;174(10):15771584.

\section{Background}

Based on early research studies, many quality and regulatory organizations have stressed the importance of assessing hospitalized patients' venous thromboembolism (VTE) risk and prophylaxing those patients at increased risk either pharmacologically or mechanically. In 2011, a meta-analysis of 40 studies of medical and stroke patients including approximately 52,000 patients failed to demonstrate a mortality benefit, showing that for every 3 pulmonary embolisms (PEs) prevented, it caused 4 major bleeding episodes per 1000 patients. ${ }^{14}$ A second study in 2011, a multicenter, randomized controlled trial with medically complex patients deemed high risk for VTE, also failed to demonstrate a mortality benefit. ${ }^{15}$ Despite these and other trials showing questionable benefit, guidelines continue to recommend that high-risk medical patients should get pharmacologic prophylaxis against VTE.

\section{Findings}

This retrospective cohort trial retrospectively evaluated a cohort of 20,794 medical patients (non-ICU) across 35 hospitals, excluding those with a Caprini score of $<2$ (ie, low risk for VTE). The authors divided the hospitals into tertiles based on adherence to VTE prophylaxis guidelines. Patients were followed to 90 days after hospitalization with telephone calls (reaching 56\%) and chart reviews (100\% reviewed) to identify clinically evident VTE events, excluding those that occurred within the first 3 days of index hospitalization. The study identified no statistically significant differences among the tertiles in terms of VTE rates, either in the hospital or at 90 days, though the overall VTE event rate was low. Interestingly, $85 \%$ of events took place postdischarge. Subgroup analyses also failed to identify a population of medical patients who benefited from prophylaxis.

\section{Cautions}

Debate about whether the Caprini risk score is the best available VTE risk scoring system exists. This study also excluded surgical and ICU patients.

\section{Implications}

This trial adds to the mounting literature suggesting that current guidelines-based pharmacologic VTE prophylaxis for medical patients may offer no clear benefit in terms of incident VTE events or mortality. Although it is not yet time to abandon VTE prophylaxis completely, this study does raise the important question of whether it is time to revisit the quality guidelines and regulatory standards around VTE prophylaxis in medical inpatients. It also highlights the difficulty in assessing medical patients for their VTE risk. Though this study is provocative and important for its real-world setting, further studies are required.

\section{OUT WITH THE OLD AND IN WITH THE NEW? SHOULD DIRECT ORAL ANTICOAGULANTS BE OUR FIRST CHOICE FOR CARING FOR PATIENTS WITH VTE AND ATRIAL FIBRILLATION?}

van Es N, Coppens M, Schulman S. et al. Direct oral anticoagulants compared with vitamin $\mathrm{K}$ antagonists for acute venous thromboembolism: evidence from phase 3 trials. Blood. 2014;124(12):1968-1975.

For patients with acute VTE, direct oral anticoagulants work as well and are safer.

\section{Background}

There have been 6 large published randomized controlled trials of direct oral anticoagulants (DOACs) versus vitamin $\mathrm{K}$ antagonists (VKAs) in patients with acute VTE. Study sizes range from approximately 2500 to over 8000 subjects. All showed no significant difference between the arms with respect to efficacy (VTE or VTE-related death) but had variable results with respect to major bleeding risk, a major concern given the nonreversibility of this group of medications. Additionally, subgroup analysis within these studies was challenging given sample size issues.

\section{Findings}

These 6 studies were combined in a meta-analysis to address the DOACs' overall efficacy and safety profile, 
as well as looking in prespecified subgroups. The meta-analysis included data from over 27,000 patients, evenly divided between DOACs (edoxaban, apixaban, rivaroxaban, and dabigatran) and VKAs, with the time in the therapeutic range (TTR) in the VKA arm being $64 \%$. Overall, the primary efficacy endpoint (VTE and VTE-related death) was similar (DOACs relative tisk $[R R]=0.90 ; 95 \%$ confidence interval $[\mathrm{CI}]: 0.77-1.06)$ but major bleeding (DOACs $\mathrm{RR}=0.61 ; 95 \% \quad \mathrm{CI}: 0.45-0.83 ; \quad \mathrm{NNT}=150)$ and combined fatal and intracranial bleeding (DOACs $\mathrm{RR}=0.37 ; 95 \%$ CI: $0.27-0.68 ; \mathrm{NNT}=314$ ) favored the DOACs. In subgroup analysis, there was no efficacy difference between the therapeutic groups in the subset specifically with DVT or with PE, or with patients weighing $>100 \mathrm{~kg}$, though safety data in these subsets were not evaluable. Patients with creatinine clearances of 30 to $49 \mathrm{~mL} / \mathrm{min}$ demonstrated similar efficacy in both treatment arms, and the safety analysis in this subset with moderate renal impairment was better in the DOAC arm. Cancer patients achieved better efficacy with similar safety with the DOACs, whereas elderly patients achieved both better safety and efficacy with DOACs.

\section{Cautions}

As yet, there are inadequate data on patients with more advanced renal failure (creatinine clearance $<30 \mathrm{~mL} / \mathrm{min}$ ) to advise using DOACs in that subset. Also, as there were no data comparing cancer patients with VTE that investigated DOACs versus low molecular weight heparins (the standard of care rather than warfarin since the CLOT [Comparison of Lowmolecular-weight heparin versus Oral anticoagulant Therapy] trial ${ }^{16}$ ), the current meta-analysis does not yet answer whether DOACs should be used in this population despite the efficacy benefit noted in the subgroup analysis.

\section{Implications}

This large meta-analysis strongly suggests we can achieve comparable treatment efficacy from the DOACs as with VKAs, with better safety profiles in patients with acute VTE. In the subset of patients with moderate renal impairment (creatinine clearance 30-49 $\mathrm{mL} / \mathrm{min})$, it appears safe and effective to choose DOACs.

\section{IN PATIENTS WITH ATRIAL FIBRILLATION, DOACs APPEAR MORE EFFECTIVE THAN VKAs WITH COMPARABLE OR BETTER SAFETY PROFILES}

Ruff CT, Guigliano RP, Braunwald E, et al. Comparison of the efficacy and safety of new oral anticoagulants with warfarin in patients with atrial fibrillation: a meta-analysis of randomized trials. Lancet. 2014;383(9921):955-962.

\section{Background}

Adding to the previously published meta-analyses of the original phase 3 randomized trials regarding the DOACs' impact on the atrial fibrillation (AF) treatment safety and efficacy literature relative to VKAs, a 2013 trial, ENGAGE AF-TIMI 48 (Effective Anticoagulation with Factor Xa Next Generation in Atrial Fibrillation-Thrombolysis in Myocardial Infarction 48 ), with edoxaban was published and warrants inclusion to have a better opportunity to glean important subgroup information. ${ }^{17}$

\section{Findings}

This meta-analysis included data on 71,683 patients, 42,411 in the DOAC arm and 29,272 in the warfarin arm, as 2 of the trials were 3 -arm studies, comparing warfarin to a high dose and a low dose of the DOAC. Meta-analyses of the 4 trials were broken down into a high-dose subset-the 2 high-dose arms and the standard doses used in the other 2 trials-and a low-dose subset-the 2 low-dose arms and the standard doses used in the other 2 trials. With respect to the efficacy endpoint (incident stroke or systemic embolization), the high-dose subset analyses of the DOACs yielded a $19 \%$ reduction $(P<0.0001 ; \mathrm{NNT}=142)$ relative to the VKAs. The safety endpoint of major bleeding in this analysis identified a $14 \%$ reduction in the DOAC group that was nonsignificant $(P=0.06)$. Within the high-dose subset, analyses favored DOACs with respect to hemorrhagic stroke $(51 \%$ reduction; $P<0.0001$; NNT $=220)$, intracranial hemorrhage $(52 \%$ reduction; $P<0.0001 ; \mathrm{NNT}=132)$, and overall mortality $(10 \%$ reduction; $P=0.0003 ; \quad \mathrm{NNT}=129$ ), whereas they increased the risk of gastrointestinal bleeding $(25 \%$ increase; $P=0.043$; $\mathrm{NNH}=185$ ). There was no significant difference between DOACs and warfarin with respect to ischemic stroke. The low-dose subset had similar overall results with even fewer hemorrhage strokes balancing a higher incidence of ischemic strokes in the DOAC arm than in warfarin. Other important subgroup analyses suggest the safety and efficacy impact of DOACs is significant for VKA-naive and experienced patients, though only statistically so for VKA-naive patients. Additionally, the anticoagulation centers included in the study that had a TTR $<66 \%$ seemed to gain a safety advantage from the DOACs, whereas both TTR groups $(<66 \%$ and $\geq 66 \%)$ appeared to achieve an efficacy benefit from DOACs.

\section{Cautions}

There are not sufficient data to suggest routinely switching patients tolerating and well managed on VKAs to DOACs for AF.

\section{Implications}

DOACs reduce stroke and systemic emboli in patients with AF without increasing intracranial bleeding or hemorrhagic stroke, though at the cost of increased 
gastrointestinal bleeding in patients on the high-dose regimens. Those patients on the low-dose regimens have even a lower hemorrhagic stroke risk, the benefit of which is negated by a higher than VKA risk of ischemic strokes. Centers with lower TTRs (and perhaps by extrapolation, those patients with more difficulty staying in the therapeutic range) may gain more benefit by switching. New patients on treatment for AF should strongly be considered for DOAC therapy as the first line.

\section{IN ELDERLY PATIENTS, THE DOACS APPEAR TO OFFER IMPROVED EFFICACY WITHOUT SACRIFICING SAFETY}

Sardar P, Chatterjee S, Chaudhari S, Lip GYH. New oral anticoagulants in elderly adults: evidence from meta-analysis of randomized trials. J Am Geriatr Soc. 2014;62(5):857-864.

\section{Background}

The prevalence of AF rises with age, as does the prevalence of malignancy, limited mobility, and other comorbidities that increase the risk for VTEs. These factors may also increase the risk of bleeding with conventional therapy with heparins and VKAs. As such, understanding the implications of using DOACs in the elderly population is important.

\section{Findings}

This meta-analysis included the elderly (age $\geq 75$ years) subset of patients from existing AF treatment and VTE treatment and prophylaxis randomized trials comparing DOACs with VKAs, low-molecular-weight heparin (LMWH), aspirin, or placebo. The primary safety outcome was major bleeding. For AF trials, the efficacy endpoint was stroke or systemic embolization, whereas in VTE trials it was VTE or VTE-related death. Authors were able to extract data on 25,031 patients across 10 trials that evaluated rivaroxaban, apixaban, and dabigatran (not edoxaban), with follow-up data ranging from 35 days to 2 years. For safety outcomes, the 2 arms showed no statistical difference (DOAC: 6.4\%; conventional therapy: 6.3\%; OR: $1.02 ; 95 \%$ CI: 0.73-1.43). For efficacy endpoints in VTE studies, DOACs were more effective $(3.7 \%$ vs 7.0\%; OR: 0.45 ; 95\% CI: 0.27-77; NNT $=30)$. For $\mathrm{AF}$, the efficacy analysis favored DOACs also $(3.3 \%$ vs $4.7 \%$; OR: $0.65 ; 95 \%$ CI: $0.48-0.87$; $\mathrm{NNT}=71$ ). When analyzed by the efficacy of the individual DOAC, rivaroxaban and apixaban both appeared to outperform the VKA/LMWH arm for both VTE and AF treatment, whereas data on dabigatran were only available for $\mathrm{AF}$, also showing an efficacy benefit. Individual DOAC analyses for safety endpoints showed all the 3 to be similar to VKA/LMWH.

\section{Cautions}

Authors note, however, that coexisting low body weight and renal insufficiency may influence dosing choices in this population. There are specific dosage recommendations in the elderly for some DOACs.

\section{Implications}

The use of DOACs in patients aged 75 years and older appears to confer a substantial efficacy advantage when used for treatment of VTE and AF patients. The safety data presented in this meta-analysis suggest that this class is comparable to VKA/LMWH medications.

\section{CHANGING INPATIENT MANAGEMENT OF SKIN INFECTIONS}

Boucher, H, Wilcox M, Talbot G, et al. Once-weekly dalbavancin versus daily conventional therapy for skin infection. N Engl J Med. 2014;370:2169-2179.

Corey G, Kabler, H, Mahra P, et al. Single-dose oritavancin in the treatment of acute bacterial skin infections. N Engl J Med. 2014;370:2180-2190.

\section{Background}

There are over 870,000 hospital admissions yearly for skin infection, making it one of most common reasons for hospitalization in the United States. ${ }^{18}$ Management often requires lengthy treatments with intravenous antibiotics, especially with the emergence of methicillin-resistant Staphylococcus aureus. Results from 2 large randomized, double-blinded, multicenter clinical trials were published looking at new onceweekly intravenous antibiotics. Dalbavancin and oritavancin are both lipoglycopeptides in the same family as vancomycin. What is unique is that their serum drug concentrations exceed the minimum inhibitor concentrations for over a week. Both drugs were compared in noninferiority trials to vancomycin. The studies had similar outcomes. The dalbavancin results are presented below.

\section{Findings}

Researchers randomized 1312 patients with significant cellulitis, large abscess, or wound infection. Patients also had fever, leukocytosis, or bandemia, and the infection had to be deemed severe enough to require a minimum of 3 days of intravenous antibiotics. The patients could not have received any prior antibiotics. Over $80 \%$ of the patients had fevers, and more than half met the criteria for systemic inflammatory response syndrome. Patients were randomized to either dalbavancin (on day 1 and day 8 ) or vancomycin every 12 hours ( $1 \mathrm{gm}$ or $15 \mathrm{mg} / \mathrm{kg}$ ), with both groups receiving placebo dosing of the other drug. The blinded physicians could decide to switch to oral agent (placebo or linezolid in the vancomycin group) anytime after day 3, and the physicians could stop antibiotics anytime after day 10. Otherwise, all patients received 14 days of antibiotics.

The FDA-approved outcome was cessation of spread of erythema at 48 to 72 hours and no fever at 3 independent readings. Results were similar in the dalbavancin 
group compared to the vancomycin-linezolid group $(79.7 \%$ vs $79.8 \%)$. Dalbavancin was deemed noninferior to vancomycin. Blinded investigator's assessment of treatment success at 2 weeks was also similar $(96 \%$ vs $96.7 \%$, respectively). More treatment-related adverse events occurred in the vancomycin-linezolid group (183 vs $139 ; P=0.02$ ) and more deaths occurred in the vancomycin group (7 vs $1 ; P=0.03$ ).

\section{Cautions}

These antibiotics have only been shown effective for complicated, acute bacterial skin infections. Their performance for other gram-positive infections is unknown. In the future, it is possible that patients with severe skin infections will receive a dose of these antibiotics on hospital day 1 and be sent home with close follow-up. However, that study has not been done yet to confirm efficacy and safety. Though the drugs appear safe, there needs to be more clinical use before they become standard of care, especially because of the long half-life. Finally, these drugs are very expensive and provide broad spectrum grampositive coverage. They are not meant for a simple cellulitis.

\section{Implications}

These 2 new once-weekly antibiotics-dalbavancin and oritavancin-are noninferior to vancomycin for acute bacterial skin infections. They provide alternative treatment choices for managing patients with significant infections requiring hospitalization. In the future, they may change the need for hospitalization of these patients or significantly reduce their length of stay. Though expensive, a significant reduction in hospitalization will offset costs.

\section{SHOULD THEY STAY OR SHOULD THEY GO? FAMILY PRESENCE DURING CPR MAY IMPROVE THE GRIEF PROCESS DURABLY}

Jabre P, Tazarourte K, Azoulay E, et al. Offering the opportunity for family to be present during cardiopulmonary resuscitation: 1 year assessment. Intensive Care Med. 2014;40:981-987.

\section{Background}

In 2013, a French study randomized adult family members of a patient undergoing cardiopulmonary resuscitation (CPR) occurring at home to either be invited to stay and watch the resuscitation or to have no specific invitation offered. ${ }^{19}$ At 90 days, this study revealed that those who were invited to watch (and $79 \%$ did) had fewer symptoms of post-traumatic stress disorder (PTSD) $(27 \%$ vs $37 \%)$ and anxiety $(15 \%$ vs $23 \%)$, though not depression, than did the group not offered the opportunity to watch (though $43 \%$ watched anyway). There were 570 subjects (family members) in the trial, of whom a greater number in the control arm declined to participate in a 90-day follow-up due to emotional distress. Notably, only
$4 \%$ of the patients in this study undergoing CPR survived to day 28. Whether the apparent positive psychological impact of the offer to watch CPR for families was durable remained in question.

\section{Findings}

The study group followed the families up to 1 year. At that time, dropout rates were similar (with the assumption, as in the prior study, that those who dropped out of either arm had PTSD symptoms). At follow-up, subjects were again assessed for PTSD, anxiety, and depression symptoms as well as for meeting criteria for having had a major depressive episode or complicated grief. Four hundred eight of the original 570 subjects were able to undergo reevaluation. The 1-year results showed the group offered the chance to watch CPR had fewer PTSD symptoms $(20 \%$ vs $32 \%)$ and depression symptoms $(10 \%$ vs $16 \%)$, as well as fewer major depressive episodes $(23 \%$ vs $31 \%)$ and less complicated grief $(21 \%$ vs $36 \%$ ) but without a durable impact on anxiety symptoms.

\section{Cautions}

The resuscitation efforts in question here occurred out of hospital (in the home). Part of the protocol for those family members observing CPR was that a clinician was assigned to stay with them and explain the resuscitation process as it occurred.

\section{Implications}

It is postulated that having the chance to observe CPR, if desired, may help the grieving process. This study clearly raises a question about the wisdom of routinely escorting patient's families out of the room during resuscitative efforts. It seems likely that the durable and important psychological effects observed in this study for family members would similarly persist in emergency department and inpatient settings, where staff can be with patients' families to talk them through the events they are witnessing. It is time to ask families if they prefer to stay and watch CPR and not automatically move them to a waiting room.

Disclosure: Nothing to report.

\section{References}

1. Reuters, T. Journals in the 2014 release of the JCR. Available at: http://scientific.thomsonreuters.com/imgblast/JCRFullCovlist-2014. pdf. Accessed August 28, 2015.

2. Jessup M. Neprilysin inhibition-a novel therapy for heart failure. $N$ Engl J Med. 2014;371(11):1062-1064.

3. Bassler D, Briel M, Montori VM, et al. Stopping randomized trials early for benefit and estimation of treatment effects: systematic review and meta-regression analysis. JAMA. 2010;303(12):1180-1187.

4. Katzberg RW, Newhouse JH. Intravenous contrast medium-induced nephrotoxicity: is the medical risk really as great as we have come to believe? Radiology 2010;256(1):21-28.

5. Persson PB, Hansell P, Liss P. Pathophysiology of contrast mediuminduced nephropathy. Kidney Int. 2005;68(1):14-22.

6. Weisbord SD, Palevsky PM. Contrast-induced acute kidney injury: short- and long-term implications. Semin Nephrol. 2011;31(3): 300-309. 
7. McDonald JS, McDonald RJ, Comin J, et al. Frequency of acute kidney injury following intravenous contrast medium administration: a systematic review and meta-analysis. Radiology. 2013;267(1):119-128.

8. Al-Aama T, Brymer C, Gutmanis I, Woolmore-Goodwin SM, Esbaugh J, Dasgupta M. Melatonin decreases delirium in elderly patients: a randomized, placebo-controlled trial. Int J Geriatr Psychiatry. 2011;26(7):687-694.

9. Elkington SG, Floch MH, Conn HO. Lactulose in the treatment of chronic portal-systemic encephalopathy. A double-blind clinical trial. N Engl J Med. 1969;281(8):408-412.

10. Hassanein, T, Blei AT, Perry W, et al. Performance of the hepatic encephalopathy scoring algorithm in a clinical trial of patients with cirrhosis and severe hepatic encephalopathy. Am J Gastroenterol. 2009;104(6):1392-1400.

11. Ge PS, Runyon BA. The changing role of beta-blocker therapy in patients with cirrhosis. J Hepatol. 2014;60(3):643-653.

12. Krag A, Wiest R, Albillos A, Gluud LL. The window hypothesis: haemodynamic and non-haemodynamic effects of beta-blockers improve survival of patients with cirrhosis during a window in the disease. Gut. 2012;61(7):967-969.

13. Ge PS, Runyon BA. When should the beta-blocker window in cirrhosis close? Gastroenterology. 2014;146(7):1597-1599.
14. Lederle FA, Zylla D, MacDonald R, Wilt TJ. Venous thromboembolism prophylaxis in hospitalized medical patients and those with stroke: a background review for an American College of Physicians Clinical Practice Guideline. Ann Intern Med. 2011;155(9):602-615.

15. Kakkar AK, Cimminiello C, Goldhaber SZ, Parakh R, Wang C, Bergmann JF; LIFENOX Investigators. Low-molecular-weight heparin and mortality in acutely ill medical patients. N Engl J Med. 2011; 365(26):2463-2472.

16. Lee AY, Levine MN, Baker RI, et al.; Randomized Comparison of Low-Molecular-Weight Heparin versus Oral Anticoagulant Therapy for the Prevention of Recurrent Venous Thromboembolism in Patients with Cancer (CLOT) Investigators. Low-molecular-weight heparin versus a coumarin for the prevention of recurrent venous thromboembolism in patients with cancer. N Engl J Med. 2003; 349(2):146-153.

17. Giugliano RP, Ruff CT, Braunwald E, et al.; ENGAGE AF-TIMI 48 Investigators. Edoxaban versus warfarin in patients with atrial fibrillation. N Engl J Med. 2013;369(22):2093-2104.

18. Chambers HF. Pharmacology and the treatment of complicated skin and skin-structure infections. N Engl J Med. 2014;370(23): 2238-2239.

19. Jabre P, Belpomme V, Azoulay E, et al. Family presence during cardiopulmonary resuscitation. N Engl J Med. 2013;368(11):1008-1018. 